\title{
Spot Weld Testing Using Active Thermography
}

\author{
by P. Myrach*, F. Jonietz*, M. Ziegler*, Hubert Suwala* and M. Rethmeier,** \\ *BAM Federal Institute for Materials Research and Testing, Unter den Eichen 87, 12205 Berlin, Germany, \\ philipp.myrach@bam.de \\ **Fraunhofer Institute for Production Systems and Design Technology, Pascalstraße 8-9, 10587 Berlin, \\ Germany
}

\begin{abstract}
The paper presents an active thermography technique for the evaluation of spot welds. The quality of spot welds is linked to the diameter of the weld nugget, which represents the mechanical connection between the two steel sheets. Since this connection also serves as a thermal bridge, distinct temperature contrasts are observed with thermography. The evaluation of these contrasts provides a measure for the spot weld diameter. The experiments were carried out in reflection as well as in transmission geometry and with flash lamp and laser illumination. A comprehensive evaluation allows to get a good discrimination for samples produced with different welding currents or of different quality respectively.
\end{abstract}

\section{Introduction}

Although spot welding is a well-established and widely applied joining technique especially in the automotive industry, a non-destructive testing method replacing the ongoing random destructive testing is still lacking. Until now, the reliability of the spot welds is usually assured by a destructive testing of random samples [1] and an accurate control of the process parameters. However, the destructive testing is expensive and time consuming and doesn't allows for checking all or at least a large number of individual spot welds. To account for these drawbacks, active thermography with optical excitation is used in this work as a non-destructive, remote and fast method to evaluate the quality of single spot welds.

\section{Principle and Setup}

Spot welding is widely used to join metal sheets. Hereby, the two metal sheets are pushed together by two copper electrodes with a constant pressure. The sheets are then joined by applying a current of a few kA for a time of 200 ms. Within this study, steel sheets (material: HX340LAD+Z100MB, HC340LA+ZE 50/50, dimension: width x length 4 $\mathrm{cm} \times 6 \mathrm{~cm}$ ) of $1 \mathrm{~mm}$ thickness were used to produce a large number of samples. A typical sample is shown in figure 1 (a).

The different samples were produced using spot welding currents ranging from $7.4 \mathrm{kA}$ to $10.4 \mathrm{kA}$. By applying different currents, the actual size of the so called weld nugget (see. Figure 1 (b)) can be altered. Hereby, smaller currents lead to a smaller diameter of the weld nugget, and since the size of the weld nugget governs the mechanical strength of the weld, it was possible to produce spot welds of different quality.

The aim of the thermographic investigation was to provide a measure for the quality of these different spot welds. In order to access this information, active thermography was performed by using either flash lamp or laser illumination. A thermographic contrast is hereby obtained by monitoring the IR radiation emitted from the surface of the sample during and after the actual illumination. The observed thermographic contrast emerges from the fact, that a heat flow through a sample is strongly influenced by the presence of the spot weld, because the weld nugget not only joins the two steel sheets mechanically, but also represents a thermal bridge between the two steel sheets. Therefore, heat can diffuse through the weld nugget, whereas it is "blocked" by the air gap (see figure 1 (b)) around the actual weld. These differences in heat flow lead to distinct temperature distributions on the sample surface that can be recorded and analysed with an IR-Camera. 

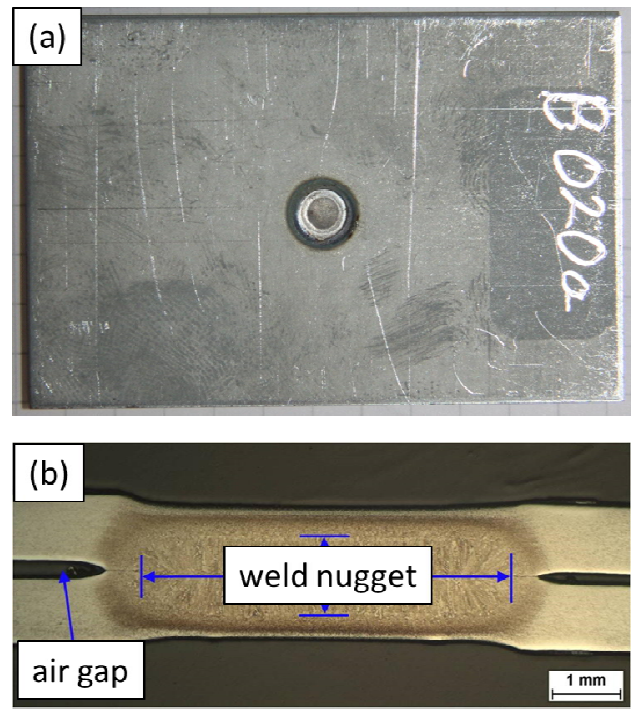

Figure 1. (a) Photography of a sample $(4.0 \mathrm{~cm} \times 6.0$ $\mathrm{cm}$ ) consisting of two $1 \mathrm{~mm}$ thick spot welded steel sheets. (b) Cut through the spot weld showing the weld nugget and the air gap between the two steel sheets.

Experiments can be carried out in two different configurations, depending on the accessibility of the actual testing problem. In transmission geometry, the IR camera as well as the heat source are at the two opposite sides of the sample [2,3,4], whereas in reflection geometry both are on the same side [4]. The setup in reflection geometry is shown in figure 2.

To record the IR-radiation emitted from the sample, an InSb focal array (512 x 640 pixel) with a spectral sensitivity ranging from $4.7 \mu \mathrm{m}-5.4 \mu \mathrm{m}$ was used. The frame rate was chosen to $40 \mathrm{~Hz}$ and the spatial resolution was 16 pixel $/ \mathrm{mm}$. The illumination of the sample surface was either done by a diode laser emitting at $950 \mathrm{~nm}$ with a maximal power of $500 \mathrm{~W}$ or a flash lamp $(6 \mathrm{~kJ})$. In order to reach sufficient temperature increase, the flash lamp had to be placed very close to the sample, which is problematic especially for the reflection geometry. The laser source, however, has the advantage, that it is in principle not restricted to specific distances to reach its maximum energy density. By choosing the optics for the laser system, an illumination even for larger ( $>$ few meters) is possible. For the experiments presented here, the optics was chosen to a focal length of $276 \mathrm{~mm}$, where the optics forms a $2 \mathrm{~cm}$ by $2 \mathrm{~cm}$ area of illumination instead of a spot.

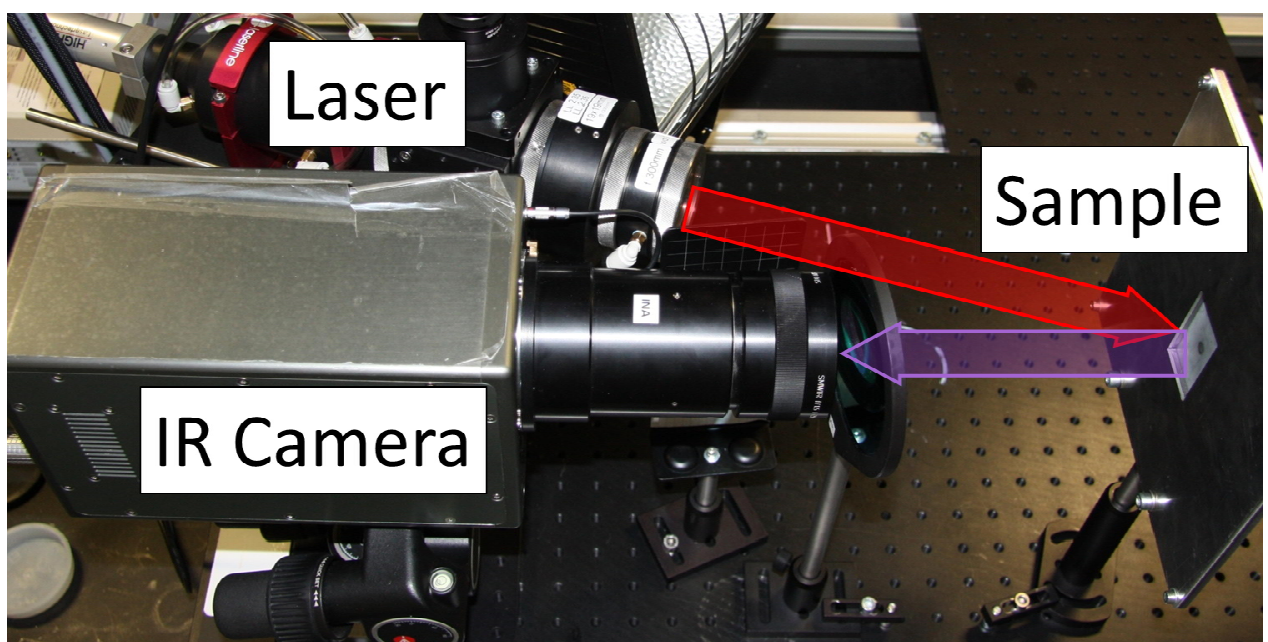

Figure. 2. Setup in reflection geometry. The IR Laser illuminates an area of $2 \mathrm{~cm} \times 2 \mathrm{~cm}$ of the sample surface and heats it up. The induced heat flow through the spot weld is then monitored by an InSb-Camera. 
In order to match the conditions of the industrial application most closely, all experiments were carried out without any coating, which is often applied in thermographic testing in order to homogenise and increase the absorption and emissivity of the sample surface. It also has to be pointed out, that working in transmission geometry is not favorable for most industrial applications, since only in rare cases both sides of the sample are accessible.

In the following, some of the results for the two setup geometries are discussed.

\subsection{Experiments in transmission geometry}

Figure 3 represents a typical thermogram as obtained in a transmission experiment with laser illumination. The laser hereby heats up the sample for 1 second with a power of $500 \mathrm{~W}$. As mentioned earlier, the contrast here is governed by the good thermal conductivity of the spot weld, such that the welded region appears as a spot of relatively higher temperature compared to the region where the air gap between the sheets restricts a heat flow. However, to gain interpretable data, a number of corrections have to be applied in order to eliminate interfering thermal contributions that would otherwise mask the signature of the weld nugget.

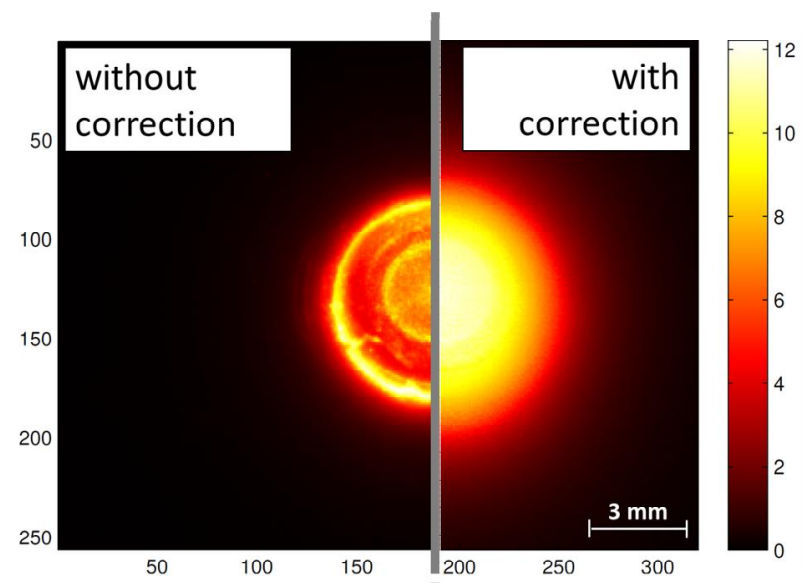

Figure 3. Thermogram of a spot welded sample produced with a current of $10.4 \mathrm{kA}$. Left: Thermogram with no emissivity correction applied. Right: Thermogram after emissivity correction.

In a first correction step, the static background radiation is removed by subtracting thermograms acquired before the illumination. The most pronounced impact on the data however is due to the inhomogeneous surface emissivity, as it is clearly visible in figure 1(a). In order to correct for this contribution, a thermogram a few seconds after the illumination is used to normalise the whole sequence. Since the heat at this point of time is already homogeneously distributed and the overall temperature of the sample is slightly increased, the observed contrast is exclusively governed by the spatial differences of the surface emissivity. Hence, the normalisation step eliminates the effect of inhomogeneous emissivity and results in a thermogram that reveals the heat distribution induced by the presence of the spot weld as shown in figure 3 (right). 

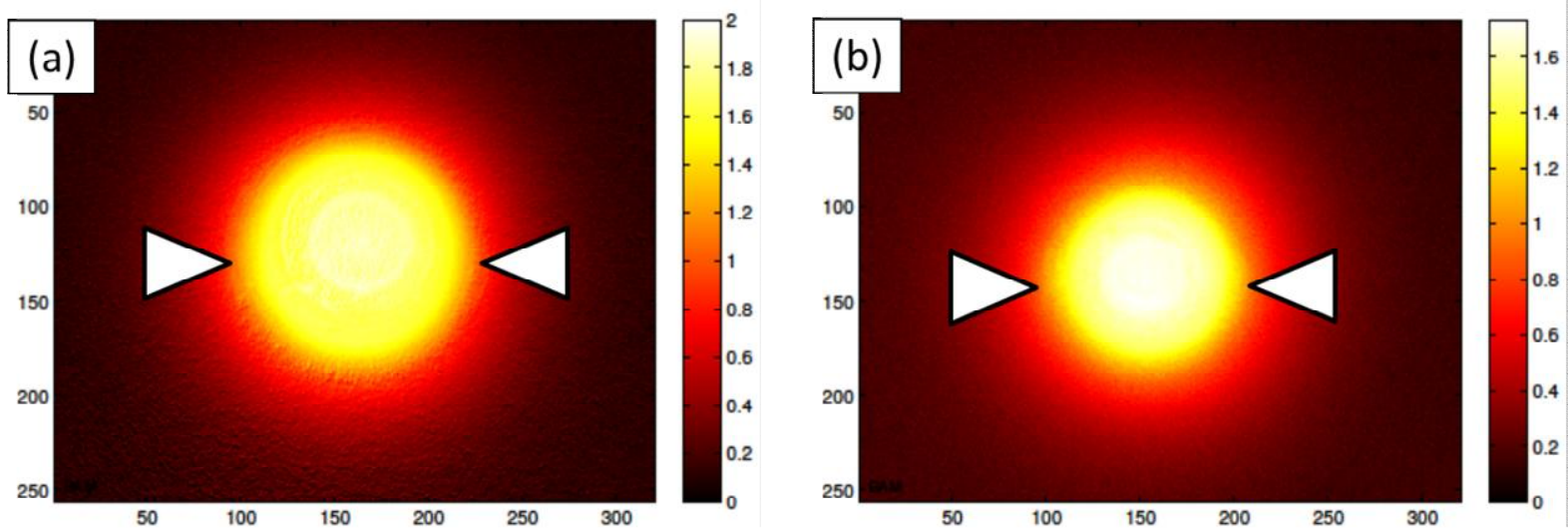

Figure 4. Corrected thermograms of a samples produced with a welding current of (a) $10.4 \mathrm{kA}$ and (b) $7.4 \mathrm{kA}$ respectively. As can be seen, measuring the FWHM provides a measure for the actual diameter of the spot weld. A smaller welding current leads to a smaller weld nugget and to a clearly smaller thermographic signature.

Figure 4 shows thermograms of two different samples that were welded with a current of $7.4 \mathrm{kA}$ and $10.4 \mathrm{kA}$, respectively. These results were obtained by illumination with a flash lamp and application of the correction described above. Comparing both thermograms clearly reveals a difference of the size of the circular warm region, which represents the actual weld nugget. It turns out that the FWHM of the thermographic signatures is a good measure for the size of the spot weld and thereby an indication of the mechanical strength of the joint.

In transmission geometry, both, laser and flash lamp illumination was sufficient to get a good discrimination for different welding currents or weld diameters respectively. However, the discrimination was best for the experiments carried out with the laser source.

\subsection{Experiments in reflection geometry}

For an industrial application, it is more important to be able to perform a thermographic testing in reflection configuration since one-sided accessibility is common. Therefore, the experiments were repeated in reflection geometry, as shown in figure 2.

Using the geometry, it turned out that a realisation with flash lamp illumination was not possible, due to the fact that the flash lamp had to be placed very close to the sample in order to achieve a sufficient irradiance, which is difficult geometrically. Furthermore, the flash lamp cools down relatively slow and this leads to reflections of infrared radiation on the sample surface that could not be eliminated by the previously described corrections. Without the correction, a reliable evaluation of the spot weld diameter was not possible.

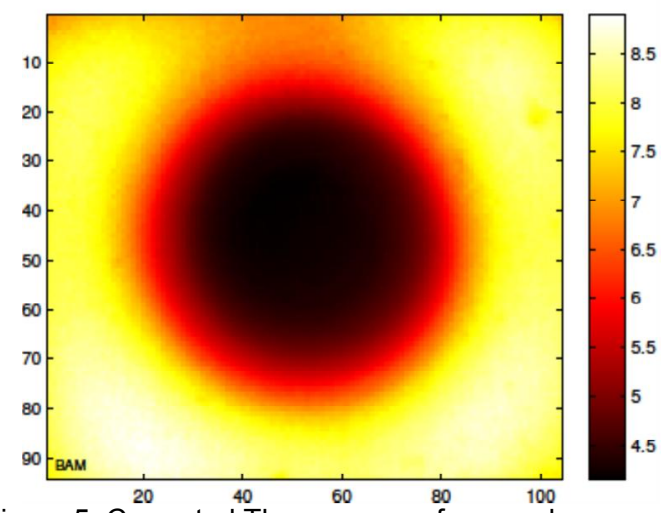

Figure 5. Corrected Thermogram of a sample produced with a welding current of $10.4 \mathrm{kA}$ and measured in reflection geometry with laser illumination.

However, using the laser illumination allows to counteract these problems. The laser can be focused over large distances and still induces high temperature contrasts. Therefore reflection experiments were carried out using a laser 
illumination of an area of $2 \mathrm{~cm} \times 2 \mathrm{~cm}$, a power of $500 \mathrm{~W}$ and pulse duration of 1 second. Since no coating (i.e. surface blackening) was applied, also for these experiments a background correction was performed. Also the correction of the inhomogeneities of the surface emissivity had to be done. In this case, this correction is possible without problems since reflected or scattered laser radiation $(950 \mathrm{~nm})$ cannot reach the IR camera $(3.7 \mu \mathrm{m}-5.4 \mu \mathrm{m})$ because of a highly reflecting band pass filter $(800 \mathrm{~nm}-1000 \mathrm{~nm})$ mounted in front of the camera (see. Figure 2).

Figure 5 shows one thermogram after all correction steps, which clearly reveals an indication of the spot weld. In this case, the observed contrast is reversed compared to the transmission experiments; because the heat can efficiently diffuse through the spot weld, and therefore the weld region of the first sheet (i.e. the illuminated one) cools down faster than the surrounding material.

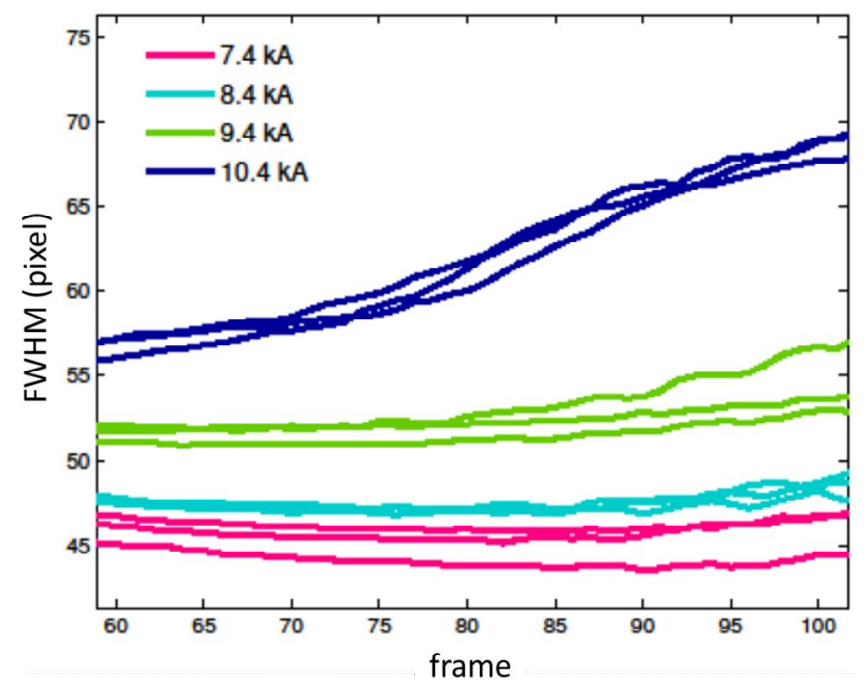

Figure 6. Diameter (FWHM) of the thermographic signature of the spot weld versus frame number (time) for different samples produced with different welding currents.

The evaluation of the spot diameter (FWHM) for samples produced with different welding currents (7.4 kA $10.4 \mathrm{kA}$ ) shows that the extracted diameter not only evolves in time (see figure 6 ), but also shows a strong correlation with the welding current. Hence, this evaluation serves as a good discrimination of the spot weld diameter and a reliable measure for the actual quality of individual joints.

\section{Conclusions}

The technique of active thermography with optical illumination is a method that can provide a measure for the quality of different spot welds. It is possible to perform these investigations for both transmission and reflection geometry. However, the one-sided evaluation could only be implemented with the laser source. This is mainly due to the low induced temperature contrast and the perturbing reflections of the radiation emitted by the flash lamp also after the initial flash.

For all presented experiments, a background correction had to be performed, which accounts for the static radiation from the environment. In addition, the differences in surface emissivity had to be corrected by a normalisation step. The application of these correction steps allowed gaining thermograms that distinctly show the thermal properties of the samples. The thermal signatures represent the diameter of the spot weld, which is a measure for the mechanical strength of the joint.

As a result, this method serves as a promising candidate for a remote, non-destructive and fast application to evaluate the quality of individual spot welds.

\section{Acknowledgement}

The IGF-project $17686 \mathrm{~N}$ of the Research Association for Steel Application - FOSTA, Sohnstraße 65, 40237 Düsseldorf, was funded via AiF by the Federal Ministry for Economic Affairs and Energy (BMWi) within the framework of the program Industrial Collective Research (IGF) on the basis of a decision of the German Bundestag. 
http://dx.doi.org/10.21611/qirt.2015.0036

\section{REFERENCES}

[1] ISO 10447, Widerstandschweißen - Schäl-, Meißel- und Keilprüfung von Widerstandspunkt- und Buckelschweißverbindungen

[2] Schlichtung, J., Brauser, S., Pepker, L., Maierhofer, C., Rethmeier, M., Kreutzbruck, M. "Thermographic testing of spot welds". NDT\&E international, 48, 23, 2012.

[3] Schlichting, J.; "Integrale Verfahren der aktiven Infrarotthermografie”; PHD-thesis at BAM Federal Institute for Materials Research and Testing, May 2012

[4] Lockheed Corporation, Adams, M.J., Crisman, E.M., "Method of and apparatus for thermographic evaluation of spot welds", US Patent 4854724, Filing date: 5. March 1986. 